\title{
¿POR QUÉ SE EXPANDE HACIA EL SUR LA DISTRIBUCIÓN GEOGRÁFICA DE TILLANDSIA RECURVATA (BROMELIACEAE)?
}

\author{
WHY IS THE GEOGRAPHIC DISTRIBUTION OF TILLANDSIA RECURVATA \\ (BROMELIACEAE) EXPANDING TO THE SOUTH?
}

\begin{abstract}
División Plantas Vasculares. Museo de La Plata. Facultad de Ciencias Naturales y Museo, Universidad Nacional de La Plata. Paseo del Bosque s/n. 1900, La Plata, Buenos Aires. CONICET.
\end{abstract}

*eguerrero@fcnym.unlp.edu.ar

Citar este artículo

APODACA, M. J. \& E. L. GUERRERO. 2019. ¿Por qué se expande hacia el sur la distribución geográfica de Tillandsia recurvata (Bromeliaceae)?. Bol. Soc. Argent. Bot. 54: 255-261.

DOI: http://dx.doi. org/10.31055/1851.2372.v54 n2.24371
Recibido: 25 Septiembre 2018 Aceptado: 14 Mayo 2019 Publicado: 30 Junio 2019 Editor: Omar Varela (D)

ISSN versión impresa 0373-580X ISSN versión on-line 1851-2372

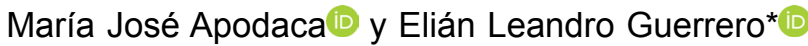

\section{SUMMARY}

Background and aims: The geographical distribution of Tillandsia recurvata ranges from the southern United States to the center of the Argentine. The aim of this work is to report the presence of this species $200 \mathrm{~km}$ south of its known distribution and to discuss the causes that may have led to this range expansion.

M \& M: Specimens were collected in different locations in the province of Buenos Aires. Herbaria collections, on-line databases and bibliography were analyzed to corroborate the taxonomic identification and the distribution limit of the species.

Results: We present novel records of Tillandsia recurvata that extend its geographic distribution $200 \mathrm{~km}$ towards the South, being the locality of Tandil the southernmost site of occurrence.

Conclusions: Because the dates of collection and observations are recent, we consider that this advance occurred in the last decades. Possible causes are discussed, such as climate change and lignification of the Pampas.

\section{KEY WORDS}

Groves, Celtis tala forest, Climate change, Epiphytes.

\section{RESUMEN}

Introducción y objetivos: la distribución geográfica de Tillandsia recurvata abarca desde el sur de Estados Unidos hasta el centro de la Argentina. El objetivo de este trabajo es reportar la presencia de esta especie $200 \mathrm{~km}$ al sur de su distribución conocida y discutir las causas que puedan haber conducido a esta expansión de rango.

M\&M: Se colectaron especímenes en diferentes localidades de la provincia de Buenos Aires. Se analizaron colecciones de herbario, bases de datos on-line y bibliografía para corroborar la identificación taxonómica y el límite de distribución de la especie.

Resultados: Se presentan registros novedosos de Tillandsia recurvata (L.) L. que amplían su distribución geográfica 200 km hacia el Sur, siendo la localidad de Tandil el sitio más austral de ocurrencia.

Discusión: Debido a que las fechas de colecta y observaciones son recientes, consideramos que este avance ocurrió en las últimas décadas. Se discuten las posibles causas, como el cambio climático y la lignificación de las Pampas.

Palabras clave

Arboledas, Bosques de Celtis tala, Cambio climático, Epífitas.

\section{INTRODUCCIÓN}

El género Tillandsia, con alrededor de 700 especies, es el género más importante de Bromeliaceae en número de especies (Barfuss et al., 2016; Gouda \& Butcher, 2017). La gran mayoría de sus especies son epífitas, presentando adaptaciones a este modo de vida como tricomas absorbentes y metabolismo CAM (metabolismo ácido de las crasuláceas).

Una especie relevante del género por su distribución geográfica y potencial utilidad para la industria médica y como bioindicadora es 
Tillandsia recurvata (L.) L. (Fig. 1). Es una de las especies de mayor distribución geográfica: al igual que T. usneoides L., abarca prácticamente toda la Región Neotropical, desde el sur de Estados Unidos hasta el centro de la República Argentina, esquivando la Cuenca Amazónica (Smith \& Downs, 1977). El límite austral de T. recurvata según Smith \& Downs (1977) y Cabrera (1968) son los bosques costeros húmedos, bosques secos de Celtis tala Gillies ex Planch., y áreas urbanas de la costa del Río de la Plata.

Dada la gran amplitud de condiciones ecológicas en las que $T$. recurvata puede habitar, se considera a esta especie como eurioica. Crece tanto epífita como epipétrica, pudiéndosela hallar en ambientes naturales creciendo sobre la copa de árboles de gran altura, cactus o sobre arbustos a menos de un metro del suelo, o en áreas urbanas sobre edificaciones y cables. Entre sus adaptaciones más notables al epifitismo, se ha comprobado que $T$. recurvata tiene bacterias endosimbiontes fijadoras de nitrógeno en el tejido vascular del tallo (Puente \& Basham, 1994), tricomas absorbentes con los que puede absorber agua de neblina (Guevara-Escobar et al., 2011) y métodos complejos de regulación de pérdida de agua por evapotranspiración asociado al metabolismo CAM (Lange \& Medina, 1979).

Es importante destacar que $T$. recurvata tiene promisorias aplicaciones médicas en el campo de la oncología (Lowe et al., 2012, 2013, 2014, 2017). Es también una planta que puede ser utilizada como bioindicadora de polución ambiental (Graciano et al., 2003; Zambrano García et al., 2009; Bermúdez \& Pignata, 2011).

De acuerdo con la historia de la vegetación bonaerense, la implantación de arboledas en los cascos de estancias resultó en la introducción involuntaria de algunas especies leñosas en los pastizales de la provincia biogeográfica Pampeana, principalmente a partir del siglo XIX (Zalba \& Villamil, 2002; Bilenca \& Miñarro, 2004). Este proceso ha transformado el ecosistema original a tal punto, que es prácticamente imposible recuperarlo sin intervención humana (Ghersa et al., 2002).

Las formaciones boscosas, aunque escasas, estaban naturalmente presentes en el centro y este de Buenos Aires con anterioridad a la colonización europea (Delucchi \& Charra, 2012). La distribución de los talares (bosques de C. tala) abarca todo el

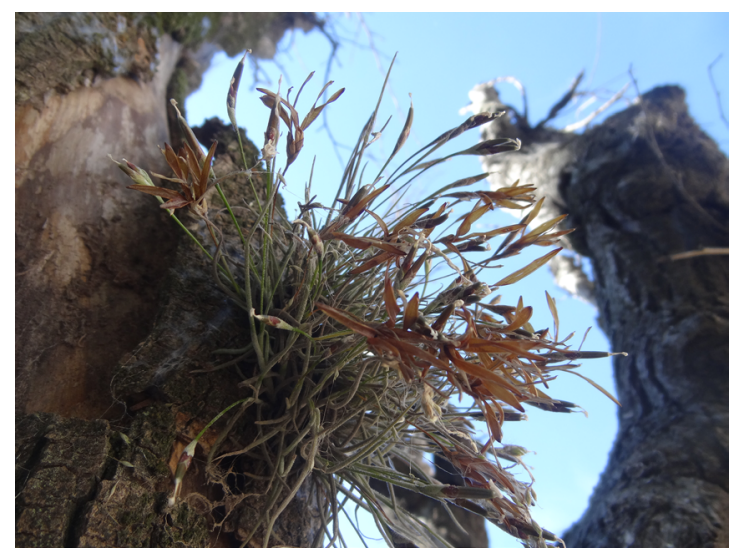

Fig. 1. Tillandsia recurvata sobre arbolado público en la Ciudad de Dolores.

borde norte y este de la provincia de Buenos Aires hasta la localidad de Mar del Plata (Parodi, 1940). Esta comunidad puede ser una vía de penetración de animales y plantas de la ecorregión Espinal hacia el sur (Agnolin et al., 2017). En las sierras del Sistema de Tandil se desarrollan arbustales de Baccharis dracunculifolia subsp. tandilensis (Speg.) Giuliano, Colletia paradoxa (Spreng.) Escal. y Dodonea viscosa Jacq. (Cabrera, 1968), $\mathrm{y}$ en los arroyos que surcan la región crecen bosques en galería de Salix humboldtiana Willd. (Zalba \& Villamil, 2002). Algunas de las especies de estos bosques y arbustales también se han expandido durante el último siglo, contribuyendo a la lignificación de las Pampas. En el centro-este de Buenos Aires se ha observado la expansión de los parches de Celtis tala y de algunas aves asociadas a estos bosques (Chimento et al., 2012). Por su parte, el arbusto Baccharis dracunculifolia subsp. tandilensis se ha extendido en las últimas décadas hacia áreas de llanura (Giuliano \& Plos, 2014).

El aumento progresivo de las precipitaciones (Berbery et al., 2006) y la temperatura en la región (Barros et al., 2015) podrían estar provocando la expansión hacia el sur de numerosos animales y plantas del norte de Buenos Aires (Guerrero \& Agnolin, 2016; Guerrero \& Cellini, 2017). En el este y sur de la provincia algunos organismos han sufrido extensiones hacia el sur de su distribución geográfica que pueden estar relacionadas con el mismo fenómeno (e.g., Farina, 2006; Zamorano \& Scillatto Yané, 2008; Doumecq-Milieu et al., 2012; Nuñez Bustos, 2016). 
En base a materiales herborizados y observaciones de campo se presenta en este trabajo la ampliación de la distribución geográfica de Tillandsia recurvata hasta Tandil, 200 kilómetros hacia el sur. Se discuten las hipótesis que podrían explicar esta extensión en la distribución: 1- la especie pasó desapercibida por los coleccionistas; 2- fue introducida involuntariamente; 3- la especie sufrió una expansión reciente en su distribución por el cambio climático o por el aumento de las arboledas implantadas en la provincia Pampeana (lignificación de las Pampas).

\section{Materiales y Métodos}

Secolectaron especímenes en diferentes localidades de la provincia de Buenos Aires (Argentina), los cuales fueron herborizados y depositados en la colección del Herbario del Museo de La Plata (LP). Los viajes de campaña se realizaron mayoritariamente en primavera y verano a localidades del este de la provincia en los partidos de Chascomús, General Lavalle, Tordillo, Dolores, Castelli, General Madariaga, La Costa, etc. Adicionalmente se examinaron los ejemplares de la colección del Herbario del Museo de La Plata (LP) y del Herbario del Museo Argentino de Ciencias Naturales (BA) para corroborar la determinación taxonómica. Los límites de distribución geográfica se establecieron mediante la bibliografía, los materiales de herbario examinados y la utilización de bases de datos on-line (https://www.gbif.org/species/search, http://www.tropicos.org/, http://www.darwin.edu. ar/proyectos/floraargentina/fa.htm, http://www. floraargentina.edu.ar/).

\section{Resultados}

Se expande la distribución geográfica de $T$. recurvata $200 \mathrm{~km}$ hacia el sur, siendo hasta ahora la ciudad de Tandil el registro más austral de la especie (Fig. 2). Existen registros de localidades intermedias entre el Río de la Plata y esta última, como ser algunos especímenes recolectados en los partidos de Chascomús y Dolores.

Material examinado. ARGENTINA. Prov. Buenos Aires: Partido de Chascomús, La Adela (-35.6857 , -57.9560 $)$, 08/12/2005, Torres Robles, Arturi y

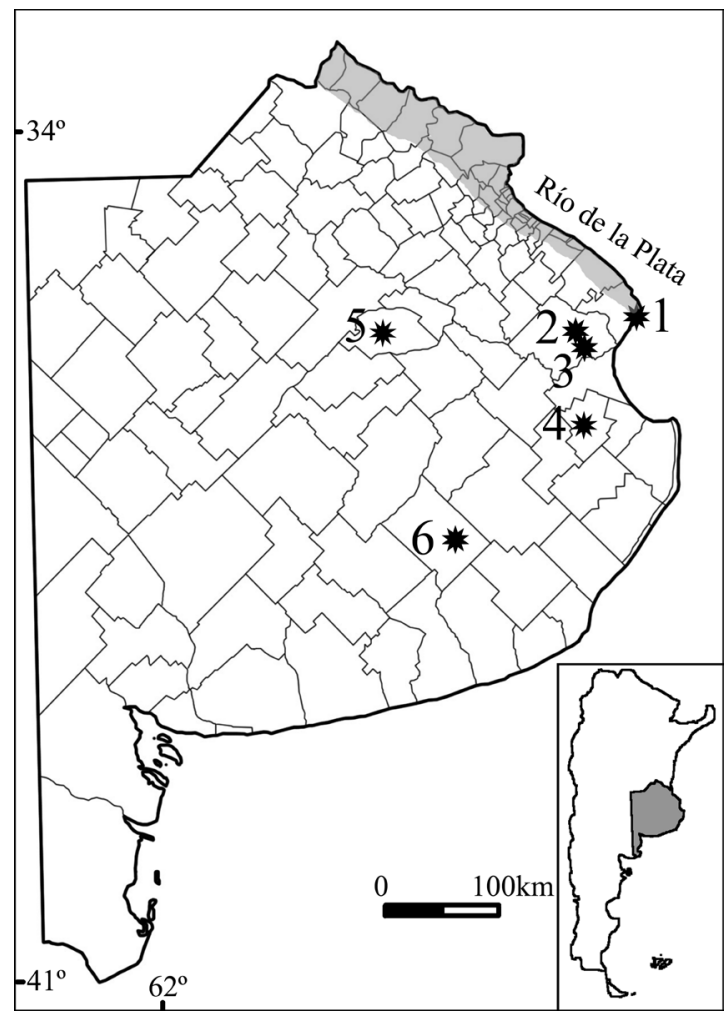

Fig. 2. Mapa de distribución de Tillandsia recurvata en la provincia de Buenos Aires. En gris la distribución conocida hasta Cabrera (1968); Localidades mencionadas en el texto= 1: Estancia Juan Gerónimo; 2: Ciudad de Chascomús y La Adela; 3: La Salada de Monasterio; 4: Saladillo; 5: Dolores; 6: Tandil.

Voglino, $N^{o} 2356$ (LP); Ciudad de Chascomús, Parque Los Libres del Sur $\left(-35.5812^{\circ},-58.0167^{\circ}\right)$, 26/08/2018, Guerrero No 723 (LP). Partido de Punta Indio, Estancia Juan Gerónimo (-35.5468 -57.2274), 26/10/2004, Torres Robles, Simón y González, $N^{o} 1775$ (LP). Partido de Dolores, Ciudad de Dolores, Plaza Castelli $\left(-36.3152^{\circ},-57.6753^{\circ}\right)$, 7/2/2019, Apodaca, Guerrero y Dosil s.n. (LP); Ciudad de Dolores, esquina de calle Buenos Aires y Junín (-36.3033 $\left.{ }^{\circ},-57.6846^{\circ}\right), 7 / 2 / 2019$, Apodaca, Guerrero y Dosil s.n. (LP); Partido de Tandil, Ruta $226,3 \mathrm{~km}$ al este de Tandil $\left(-37.3490^{\circ},-59.0529^{\circ}\right)$, 01/08/2017, Apodaca s.n. (LP).

Además de los materiales herborizados, la especie fue observada en: Partido de Chascomús, 
Laguna La Salada de Monasterio (-35.7751 ${ }^{\circ}$, $-57.8462^{\circ}$; E.L. Guerrero obs. pers.); Partido de Saladillo, Ciudad de Saladillo, Plaza 25 de Mayo $\left(-35.6389^{\circ},-59.7789^{\circ}\right.$; A.L. Gaddi com. pers.).

En las localidades mencionadas la especie es abundante sobre la arboleda pública y el tendido eléctrico de las ciudades. También crece sobre bosques nativos de Celtis tala en las localidades de Juan Gerónimo y Laguna La Salada de Monasterio.

\section{Discusión}

Si bien es imposible descartar por completo la hipótesis de que Tillandsia recurvata haya pasado desapercibida por los coleccionistas, el hecho de que varios botánicos y ecólogos hayan trabajado detalladamente en la Depresión del Río Salado, la Costa Atlántica y las Sierras de Tandilia (e.g., Cabrera, 1941; Vervoorst, 1967; Frangi, 1975; Faggi \& Cagnoni, 1991), sumado a los actuales hallazgos de $T$. recurvata hacia el sur de su rango geográfico conocido, dan mayor sustento a la hipótesis de una expansión reciente de la distribución de la especie. Además, la composición florística de la provincia de Buenos Aires fue estudiada de una manera minuciosa para la creación de la Flora de la Provincia de Buenos Aires (Cabrera, 1963-1970).

Podría ser factible que Tillandsia recurvata haya sido introducida involuntariamente siendo transportada sobre árboles que fueron cultivados en el área de distribución "original” de la epífita y luego plantados en el centro de Buenos Aires. Una explicación como esta fue postulada para explicar la presencia del helecho epífito Microgramma vaccinifolia (Langsd. \& Fisch.) Copel. en el norte de Buenos Aires (Guerrero \& Delucchi, 2018). Esta hipótesis es poco probable para el caso de $T$. recurvata, debido a que estaríamos hablando de numerosas introducciones en distintas localidades.

El desplazamiento hacia el sur en las isohietas entre 1950-1969 y 1980-1999 alcanzó unos 200 km en la provincia de Buenos Aires (Sierra \& Pérez 2006; Berbery et al., 2006). Dadas las tendencias de cambio observadas en el clima de la región, se esperaría que muchas de las especies subtropicales cuyos límites de distribución geográfica alcanzaban en el pasado la costa del Río de la Plata, por donde pasaba la isohieta de $1000 \mathrm{~mm}$ anuales hasta mediados del siglo XX, alcancen el interior de la provincia si sus medios de dispersión lo permiten. Un desplazamiento de norte a sur en la distribución de un grupo de especies heterogéneo ecológica- y taxonómicamente implica un grado de apoyo fuerte a la hipótesis de que el cambio climático modifica los límites de distribución. Por lo contrario, si un grupo de especies modificaran sus distribuciones tanto en dicha dirección como en la dirección contraria, se debería recurrir a otra fuente de explicación, por ejemplo, una detonante antrópica, como la lignificación de las Pampas de Buenos Aires. De ser la lignificación de las Pampas el causante principal de las modificaciones podría esperarse que, por ejemplo, Tillandsia minutiflora Donadío, T. pedicellata (Mez) A. Cast., T. gilliesii Baker y $T$. retorta Griseb. (especies epífitas que habitan las Sierras Australes de Buenos Aires) se expandan hacia el norte.

Como fue mencionado, en tan solo un siglo, el pastizal que caracterizaba las Pampas de Buenos Aires prácticamente cambió su fisonomía a una planicie arbolada. Estos árboles, tanto los exóticos como los nativos, sirven de soporte a Tillandsia recurvata, por lo cual es posible que la expansión de esta especie tenga alguna relación con este factor. Sin embargo, el hecho de que existían bosques y arbustales nativos con anterioridad a la época en que inicia la lignificación de las Pampas, nos lleva a preguntarnos por qué $T$. recurvata no se extendía hasta Tandil siguiendo los bosques nativos. Por otra parte, no se registran especies del sur de las Pampas que avancen hacia el norte, con la excepción del arbusto Baccharis dracunculifolia subsp. tandilensis. Por estas razones consideramos que, aunque la lignificación de las Pampas puede ayudar a $T$. recurvata a extenderse hacia el sur, posiblemente el detonante de este cambio ha sido otro factor.

El caso de Tillandsia recurvata es llamativamente similar a lo que ha ocurrido en la misma región con insectos (Farina, 2006; Nuñez Bustos, 2016), anfibios (Agostini \& Roesler, 2011; Agostini et al., 2016), aves (Doiny Cabré, 2006; MacLean \& Celsi, 2010) y mamíferos (Zamorano \& Scillato Yané, 2008; Doumecq Millieu et al., 2012). También se halló una extensión similar en T. aeranthos (Loisel.) L.B.Sm. (ELG en prep.). Es difícil explicar mediante el azar que repentinamente animales y plantas hayan logrado dispersarse en la misma dirección en el mismo lapso temporal. Esto 


\section{J. Apodaca y E. L. Guerrero - Expansión geográfica en Tillandsia recurvata}

se explica con mayor simplicidad epistemológica (economía de términos trascendentes) y semántica (menor cantidad de supuestos ad-hoc) si se postula que se ha perdido una barrera biogeográfica común a todos estos taxa.

Farina (2006) halló la mariposa Morpho epistrophus (Lepidoptera) $200 \mathrm{~km}$ más al sur de su distribución conocida en Buenos Aires. Este autor planteó que, ya que Scutia buxifolia Reissek, la planta nutricia de M. epistrophus, habitaba la región desde antaño, el avance hacia el sur de este lepidóptero respondía a las variaciones climáticas recientes, que alcanzan la misma magnitud. Con Tillandsia recurvata el caso es igual, los forófitos estaban presentes desde antaño, pero la epífita estaría avanzando hacia el sur en las últimas décadas. La especie $T$. recurvata podría estar siendo beneficiada por la creciente humedad de la región y la reducción en la cantidad y duración de las heladas. De ser así se podría esperar que, en las siguientes décadas, otras especies del noreste de la provincia colonicen localidades cada vez más australes.

\section{Contribución de los AUtores}

Ambos autores han realizado conjuntamente y a partes iguales la colecta de datos, su interpretación y redacción del manuscrito.

\section{Agradecimientos}

Agradecemos a Florencia Dosil por las fotos y la asistencia en el campo. A Jorge V. Crisci por sus comentarios. El estudio fue financiado por CONICET (PIP0446).

\section{Bibliografía}

AGNOLIN, F., S. LUCERO, N. CHIMENTO \& E. L. GUERRERO. 2017. Mamíferos terrestres de la Costa Atlántica de Buenos Aires. En CELSI, C. \& J. ATHOR (eds.), La costa Atlántica de Buenos Aires - Naturaleza y patrimonio cultural, pp.139180. Vázquez Mazini - Fundación Félix de Azara, Buenos Aires.

AGOSTINI, M. G., \& ROESLER, I. 2011. Amphibia, Anura, Hylidae, Scinax granulatus (Peters, 1871): distribution extension in central Argentina. Check List 7: 112-113.

AGOSTINI, M. G., SAIBENE, P. E., ROESLER, C. I., \& BILENCA, D. N. 2016. Amphibians of northwestern Buenos Aires province, Argentina: checklist, range extensions and comments on conservation. Check List 12: 1998.

http://dx.doi.org/10.15560/12.6.1998

BARFUSS, M. H. J., TILL W, E. M. C. LEME, J. P. PINZÓN, J. M. MANZANARES, H. HALBRITTER, R. SAMUEL \& G. K. BROWN. 2016. Taxonomic revision of Bromeliaceae subfam. Tillandsioideae based on a multi-locus DNA sequence phylogeny and morphology. Phytotaxa 279: 1-97.

http://dx.doi.org/10.11646/phytotaxa.279.1.1

BARROS, V.R., J.A. BONINSEGNA, I.A. CAMILLONI, M. CHIDIAK, G.O. MAGRÍN \& M. RUSTICUCCI. 2015. Climate change in Argentina: trends, projections, impacts and adaptation. WIREs Climate Change 6: 151-169. http://dx.doi.org/10.1002/wcc.316

BERBERY, E. H., M. DOYLE \& V. BARROS. 2006. Tendencias regionales en la precipitación. En: BARROS, V., R. CLARKE \& P. SILVA DÍAS (eds.), El cambio climático en la Cuenca del Plata, pp. 67-79. CONICET, Buenos Aires.

BERMUDEZ, G. M. \& M. L. PIGNATA. 2011. Antioxidant response of three Tillandsia species transplanted to urban, agricultural, and industrial areas. Arch. environ. Contam. Toxicol. 61: 401-413.

BILENCA, D. \& F. MIÑARRO (eds.). 2004. Identificación de áreas valiosas de pastizal. En las Pampas y campos de Argentina, Uruguay y sur de Brasil. Fundación Vida Silvestre, Buenos Aires.

CABRERA, A. L. (ed.) 1963-1970. Flora de la provincia de Buenos Aires. Instituto Nacional de Tecnología Agropecuaria, Buenos Aires.

CABRERA, A. L. 1941. Las comunidades vegetales de las dunas costaneras de la Provincia de Buenos Aires. DAGI Publicaciones técnicas 1:5-44.

CABRERA, A. L. 1968. Bromeliaceae. En: A. L. CABRERA (ed.), Flora de la Provincia de Buenos Aires, parte 1, pp. 448-459. Instituto Nacional de Tecnología Agropecuaria, Buenos Aires.

CHIMENTO, N., F. AGNOLIN, E. L. GUERRERO, A. M. LÓPEZ \& R. LUCERO. 2012. Nuevos registros de aves y consideraciones sobre la extensión geográfica de los talares al sur de la provincia de Buenos Aires. Nótulas Faunísticas 89: 1-12. 
DELUCCHI, G., \& CHARRA, G. R. 2012. La flora $\mathrm{y}$ vegetación pampeanos vistas por los cronistas y viajeros de los siglos XVIII y XIX. Historia Natural, tercera serie 2: 73-83.

DOINY CABRÉ, P. C. 2006. Aves nuevas o poco registradas para Sierra de la Ventana, provincia de Buenos Aires. Argentina. Revista Nuestras Aves 52: 21-23.

DOUMECQ MILIEU, R. E., A. MORICI \& N. A. NIGRO. 2012. Ampliación de la distribución austral del carpincho (Hydrochoerus hydrochaeris) en la provincia de Buenos Aires, Argentina. Nótulas Faunísticas 92: 1-10.

FAGGI, A. M. \& M. CAGNONI, 1991. La vegetación de Punta Rasa (Pcia. de Buenos Aires. Argentina). Parodiana 6: 363-374.

FARINA, J. L. 2006. Insectos asociados al tala (Celtis tala), en el límite sur del espinal. En: MÉRIDA, E. \& J. ATHOR (eds.), Talares bonaerenses y su conservación, pp. 166-172. Fundación de Historia Natural Félix de Azara, Buenos Aires.

FRANGI, J. 1975. Sinopsis de las comunidades vegetales y el medio de las sierras de Tandil (provincia de Buenos Aires). Bol. Soc. Argent. Bot. 16: 293-318.

GHERSA, C. M., E. DE LA FUENTE, S. SUAREZ \& R. J. LEON. 2002. Woody species invasion in the Rolling Pampa grasslands, Argentina. Agr. Ecosyst. Environ. 88: 271-278.

GIULIANO, D. A., \& PLOS, A. (2014). Baccharis L. En M. M. PONCE \& M. D. ARANA MD (coord.), F. O. ZULOAGA \& M. J. BELGRANO (eds.) Flora Vascular de la República Argentina. Volumen 7, Dicotyledoneae, Asteraceae: Anthemideae a Gnaphalieae, pp. 43-123. IBODA, CONICET, San Isidro, Argentina.

GOUDA, E. J. \& D. BUTCHER. 2017. Continuously updated. A List of Accepted Bromeliaceae Names. Utrecht, University Botanic Gardens [online]. Disponible en: http://bromNames.florapix.nl. [Acceso: 25 de septiembre 2018].

GRACIANO, C., L. V. FERNÁNDEZ \& D. O. CALDIZ. 2003. Tillandsia recurvata $\mathrm{L}$. as a bioindicator of sulfur atmospheric pollution. Ecol. Aust. 13: 3-14.

GUERRERO, E. L. \& F. L. AGNOLIN. 2016. Recent changes in plant and animal distribution in the southern extreme of the Paranaense biogeographical province (northeastern Buenos Aires province, Argentina): ecological responses to climate change? Rev. Mus. Argentino Cienc. Nat., n.s. 18: 9-30.
GUERRERO E. L. \& J. M. CELLINI. 2017. Corrimiento del límite austral de distribución geográfica en tres especies del género Pleopeltis (Polypodiaceae) en la provincia de Buenos Aires (República Argentina) y su posible relación con el cambio climático. Cuadernos de Investigación UNED 9: 51-58.

GUERRERO E. L. \& G. DELUCCHI. 2018. Microgramma vaccinifolia en la provincia de Buenos Aires, Argentina. Historia Natural. tercera serie 8: 105-108.

GUEVARA-ESCOBAR, A., M. CERVANTESJIMÉNEZ, H. SUZÁN-AZPIRI, E. GONZÁLEZSOSA, L. HERNÁNDEZ-SANDOVAL, G. MALDA-BARRERA, \& M. MARTÍNEZ-DÍAZ. 2011. Fog interception by Ball moss (Tillandsia recurvata). Hydrol. Earth Syst. Sc. 15: 2509-2518.

LANGE, O. L. \& E. MEDINA. 1979. Stomata of the CAM plant Tillandsia recurvata respond directly to humidity. Oecol. 40: 357-363.

LOWE, N., J. TOYANG, C. T. WATSON, K. N. AYEAH \& J. BRYANT. 2014. Antileukemic Activity of Tillandsia recurvata and some of its Cycloartanes. Anticancer Res. 34: 3505-3509.

LOWE, N., J. TOYANG, C. T. WATSON, K. N. AYEAH \& J. BRYANT. 2017. HLBT-100: a highly potent anti-cancer flavanone from Tillandsia recurvata (L.) L. Cancer Cell Int. 17: 38.

LOWE, H. I. C., C. T. WATSON, S. BADAL, E. N. ATEH, N. J. TOYANG \& J. BRYANT. 2012. Antiangiogenic properties of the Jamaican ball moss (Tillandsia recurvata L.). Int. Res. J. Biol. Sci. 1: 73-76.

LOWE, N., J. TOYANG \& J. BRYANT. 2013. In vitro and In vivo anti-cancer effects of Tillandsia recurvata (Ball Moss) from Jamaica. West Indian Med. J. 62: 177-180.

MAC-LEAN, H.D. \& C. E. CELSI. 2010. El Mirasol Grande Botaurus pinnatus en el sur de la provincia de Buenos Aires. Cotinga 33: 99-100.

NÚÑEZ BUSTOS, E.O. 2016. Mariposas diurnas (Lepidoptera: Papilionoidea \& Hesperioidea) de la Costa Atlántica Bonaerense, provincia de Buenos Aires, Argentina. En: ATHOR, J. \& CELSI, C.E. (eds.). La Costa Atlántica de Buenos Aires, naturaleza y patrimonio cultural, pp. 270-280. Fundación de Historia Natural Félix de Azara, Ciudad Autónoma de Buenos Aires.

PARODI, L. R. 1940. La distribución geográfica de los talares en la Provincia de Buenos Aires. Darwiniana 4: 33-56. 


\section{J. Apodaca y E. L. Guerrero - Expansión geográfica en Tillandsia recurvata}

PUENTE, M. E. \& Y. BASHAN. 1994. The desert epiphyte Tillandsia recurvata harbours the nitrogen-fixing bacterium Pseudomonas stutzeri. Can. J. Bot. 72: 406-408.

SMITH, L.B. \& R. J. DOWNS. 1977. Tillandsioideae (Bromeliaceae). Flora Neotropica 14: 663?1492.

SIERRA, E. M., \& S. P. PÉREZ. 2006. Tendencias del régimen de precipitación y el manejo sustentable de los agroecosistemas: estudio de un caso en el noroeste de la provincia de Buenos Aires, Argentina. Revista de Climatología 6: 1-12.

VERVOORST, F. B. 1967. Las comunidades vegetales de la Depresión del Salado. La Vegetación de la República Argentina, Serie Fitogeográfica $\mathrm{N}^{\circ} 7$. INTA, Buenos Aires.
ZALBA, S. M., \& C. B. VILLAMIL. 2002. Woody plant invasion in relictual grasslands. Biological Invasions 4: 55-72.

ZAMBRANO GARCÍA, A., C. MEDINA COYOTZIN, A. ROJAS AMARO, D. LÓPEZ VENERONI, L. CHANG MARTÍNEZ, \& G. SOSA IGLESIAS. 2009. Distribution and sources of bioaccumulative air pollutants at Mezquital Valley, Mexico, as reflected by the atmospheric plant Tillandsia recurvata L. Atmos. Chem. Phys. 9: 6479-6494.

ZAMORANO, M. \& G.J. SCILLATO-YANÉ. 2008. Registro de Dasypus (Dasypus) novemcinctus (Mammalia, Dasypodidae) en el sudoeste de la provincia de Buenos Aires, Argentina. BioScriba 1: 17-26. 
